\title{
ARTICLE Schizophrenia-like reduced sensorimotor gating in intact inbred and outbred rats is associated with decreased medial prefrontal cortex activity and volume
}

\author{
Carles Tapias-Espinosa (iD) ${ }^{1}$, Cristóbal Río-Álamos ${ }^{2}$, Ana Sánchez-González ${ }^{1}$, Ignasi Oliveras ${ }^{1}$, Daniel Sampedro-Viana ${ }^{1}$, \\ Maria del Mar Castillo-Ruiz ${ }^{3}$, Toni Cañete ${ }^{1}$, Adolf Tobeña ${ }^{1}$ and Alberto Fernández-Teruel ${ }^{1}$
}

Prepulse inhibition (PPI) of startle response is a measure of sensorimotor gating that is impaired in schizophrenia and in many other clinical conditions. Rat models using pharmacological or surgical strategies reveal that PPI is modulated by the cortico-striatalpallido-thalamic (CSPT) circuit. Here, we explore whether spontaneous variation in PPI in intact inbred and outbred rats is associated with functional and structural differences in the CSPT circuit. Inbred Roman High-(RHA) and Low-avoidance (RLA) and outbred heterogeneous stock (HS) rats were assessed for PPI, brain activity, and brain volume. Brain activity was assessed by c-Fos expression and brain volume by magnetic resonance imaging. Relevant structures of the CSPT circuit were evaluated, such as the medial prefrontal cortex (mPFC), cingulate cortex, hippocampus (HPC), amygdala, nucleus accumbens (NAc), and dorsal striatum. RHA showed lower PPI than RLA rats, while HS rats were stratified by their PPI levels in three groups. Reduced PPI was accompanied by decreased mPFC activity in Roman and HS rats and increased NAc shell activity in HS rats. Low PPI was also associated with decreased mPFC and HPC volumes in Roman and HS rats. This study reports a consistent relationship between decreased function and volume of the MPFC and spontaneous low-PPI levels in inbred and outbred intact rats. Moreover, our findings suggest that, apart from a hypoactive and smaller mPFC, a hyperactive NAc and smaller HPC may underlie reduced PPI levels. Our results support the notion that sensorimotor gating is modulated by forebrain structures and highlight the importance of the mPFC in its regulation.

Neuropsychopharmacology (2019) 44:1975-1984; https://doi.org/10.1038/s41386-019-0392-x

\section{INTRODUCTION}

Prepulse inhibition (PPI) of the startle response is a measure of sensorimotor gating, in which the magnitude of a startle stimulus is attenuated by the presence of a pre-stimulus of lower intensity [1]. PPI impairments are present in several neuropsychiatric disorders [2], including schizophrenia [3, 4]. In this sense, studying the neural mechanisms involved in PPI is relevant for progress in our knowledge of the neurobiological basis of schizophreniarelated features [5].

Rodent studies reveal that PPI is modulated by the corticostriatal-pallido-thalamic (CSPT) circuit involving the prefrontal cortex, thalamus, hippocampus (HPC), amygdala, nucleus accumbens (NAC, via ventral pallidum), and dorsal striatum (via ventral pallidum) efferents to the pedunculopontine nucleus [6-8]. In particular, rat models involving brain alterations have mostly focused on the medial prefrontal cortex (mPFC), HPC and NAc. As shown in these studies, PPI is reduced by treatments that either decrease or increase activity in the MPFC [9-12], HPC [5, 13-18], and NAc [19-23]. Moreover, several studies using neurodevelopmental models of schizophrenia report PPI deficits paralleled by mPFC and HPC abnormalities, such as models of prenatal administration of phencyclidine [24], prenatal LPS [25], or isolation rearing $[26,27]$. Additionally, electrical stimulation of the NAc alleviates PPI deficits in poly I:C offspring $[28,29]$, while it disrupts PPI in control-saline offspring. This evidence, derived from rat models involving neural manipulations, is generally consistent with the idea that alterations in the "mPFC-HPC-NAc" circuit [13] play a role in PPI deficits and other cognitive impairments found in schizophrenic patients [8,30-33].

Here, we explore whether spontaneous variation in PPI in intact inbred and outbred rats is associated with functional and structural differences in the CSPT circuit. Thus, we evaluated neuronal activity (through c-Fos expression) and volume (through structural magnetic resonance imaging-MRI) of relevant regions of the CSPT circuit, such as the MPFC, cingulate cortex $(\mathrm{Cg}), \mathrm{HPC}$, amygdala, NAc, and dorsal striatum, in intact rats displaying spontaneous differences in PPI, i.e., the Roman rats and the outbred heterogeneous rat stock.

The inbred Roman high- (RHA) and low-avoidance (RLA) rats were bidirectionally selected for rapid vs. non-acquisition of the two-way active avoidance task, respectively [34, 35]. Interestingly, compared with their RLA counterparts, RHA rats show spontaneous reductions in $\mathrm{PPI}$, working memory [36] and latent inhibition [37]. These divergent behavioral profiles, together with differences in dopaminergic [38, 39], serotoninergic, and glutamatergic systems [40-42], suggest that RHA rats may be a valid

\footnotetext{
'Department of Psychiatry \& Forensic Medicine, Institute of Neurosciences, Universitat Autònoma de Barcelona, Bellaterra, 08193 Barcelona, Spain; ${ }^{2}$ Department of Psychology, Austral University of Chile, Valdivia, Chile and ${ }^{3}$ Histology lab, Institute of Neurosciences, Universitat Autònoma de Barcelona, Bellaterra, 08193 Barcelona, Spain Correspondence: Carles Tapias-Espinosa (carles.tapias@uab.cat) or Alberto Fernández-Teruel (albert.fernandez.teruel@uab.cat)
} 
model of schizophrenia-related features. On the other hand, the outbred rat heterogeneous stock (hereafter, HS rats) was developed from a crossing of 8 inbred strains to obtain a stock of rats as heterogeneous as possible [43]. Their genetic heterogeneity makes them a unique tool to study the neurobiological/ genetic basis of normal and abnormal complex traits [44, 45]. Previous studies suggest that HS rats stratified by low-PPI may constitute a putative model of some schizophrenia-relevant traits $[36,46,47]$.

As reviewed above, $\mathrm{PPI}$ is impaired after several alterations that can either increase or decrease activity in the MPFC, HPC and NAc. Thus, we hypothesized that differences in PPI would be paralleled by differences in activity and/or volume within these regions, although the direction of these differences was difficult to predict.

\section{MATERIALS AND METHODS}

Subjects

We used male Roman $(\mathrm{RHA}=28 ; \mathrm{RLA}=28)$ and HS rats $(n=100)$ from our permanent colony (Dept. Psychiatry and Forensic Medicine, Universitat Autònoma de Barcelona). They were aged 3-4 months, weighing $250-350 \mathrm{~g}$. They were housed in pairs in macrolon cages $(50 \times 25 \times 14 \mathrm{~cm})$ and maintained with food and water ad libitum, maintained under a 12:12 h light-dark cycle (lights on at 08:00 a.m.) and with controlled temperature $\left(22 \pm 2{ }^{\circ} \mathrm{C}\right)$ and humidity $(50-70 \%)$.

Experimental procedures

All behavioral and MRI testing was carried out during the light cycle between 09:00-14:00 h. Experiments were performed in accordance with the Spanish legislation on "Protection of Animals
Used for Experimental and Other Scientific Purposes" and the European Communities Council Directive (2010/63/EU) on this subject. Every effort was made to minimize any suffering of the animals used in this study.

See experimental overview in Fig. 1.

Prepulse inhibition (PPI)

PPI was conducted in four sound attenuated boxes (SR-Lab Startle Response System, San Diego Instruments, US), as previously described [47]. Briefly, animals were individually located in an acrylic cylinder, which was situated in a dimly illuminated box and on the top of a platform with a sensor that detects the strength made by the rat in each trial. Noise bursts were presented via a speaker mounted $15 \mathrm{~cm}$ above the cylinder. After $5 \mathrm{~min}$ of habituation, 10 "pulse-alone" trials $(105 \mathrm{~dB}(\mathrm{~A}), \mathrm{SPL}, 40 \mathrm{~ms})$ were delivered in order to obtain a stable baseline of startle (BL1_Startle). Next, each one of the six types of trials were randomly administered ten times (60 trials in total): (i) Pulse-alone trials $(105 \mathrm{~dB}(\mathrm{~A}), \mathrm{SPL}, 40 \mathrm{~ms} ; \mathrm{BL2}$ _Startle; used to calculate the percentage of PPI); (ii) prepulses of $65 / 70 / 75 / 80 \mathrm{~dB}(A)$, SPL (20 ms) followed by the pulse stimulus $(105 \mathrm{~dB}(\mathrm{~A}), \mathrm{SPL}, 40 \mathrm{~ms})$ with an inter-stimulus interval of $100 \mathrm{~ms}$; or (iii) no-stimulus trials (background noise of $55 \mathrm{~dB}$ ). The interval between trials was $15 \mathrm{~s}$ (range 10-20 s). The \%PPI for each prepulse intensity was calculated by applying the following formula: \%PPI $=[100-$ (startle amplitude on prepulse trials / startle amplitude on "BL2_Startle" pulse-alone trials $\times 100)]$.

HS rats were stratified by their average \%PPI at prepulse intensities 65 and $70 \mathrm{~dB}(\mathrm{~A})$. Lower prepulse intensities, which are closer to the lowest threshold, are known to elicit lower levels of PPI [5]. Therefore, these prepulse intensities may be more sensitive

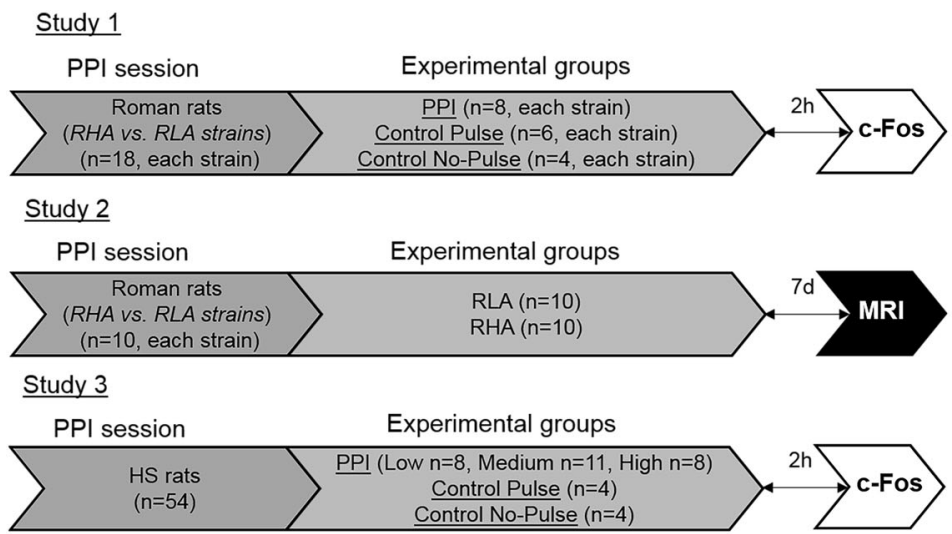

\section{Study 4}

PPI session 1

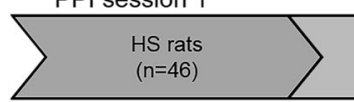

Experimental groups

PPI session 2

Low-PPI $(n=7)$
Medium-PPI $(n=12)$
High-PPI $(n=9)$
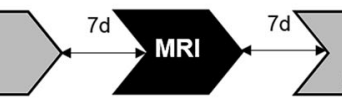

PPI (Low $n=7$, Medium $n=12$, High $n=9$ )

Control Pulse ( $n=4$, underwent PPI 1)

ontrol No-Pulse ( $n=4$, underwent PPI 1 )

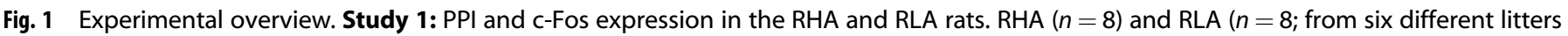

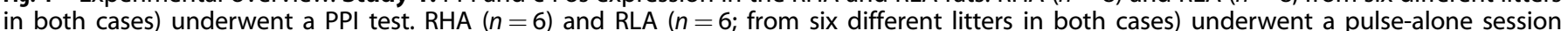

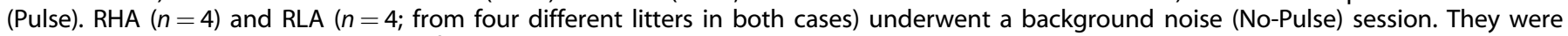

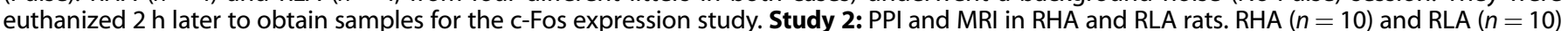

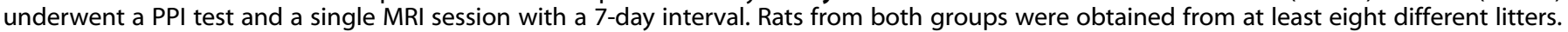

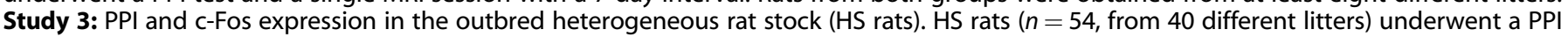

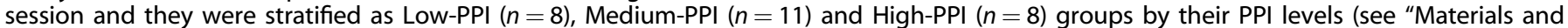

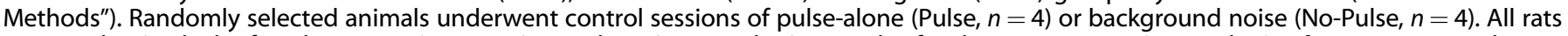

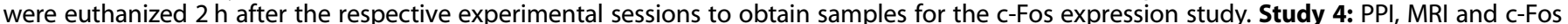

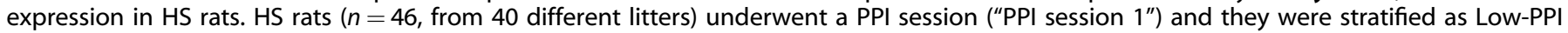

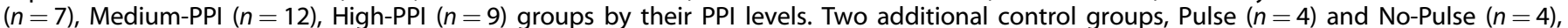

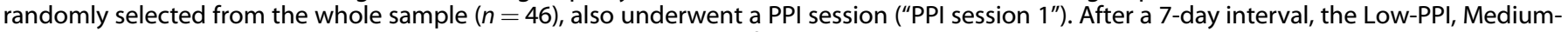

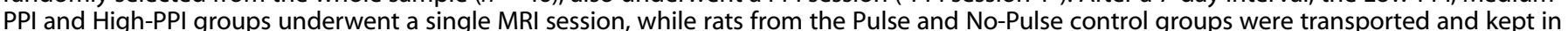

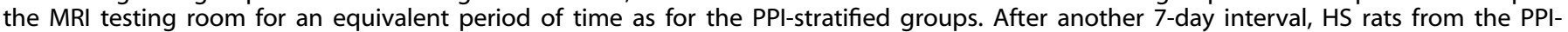

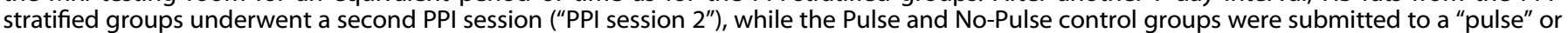

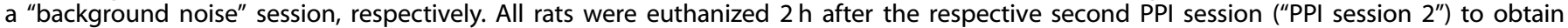
samples for the c-Fos expression study. See variable definitions and further details in "Materials and Methods" section 
to detect differences in PPI. For both experiments with HS rats (studies 3 and 4, see Fig. 1), subjects that were one standard deviation below or above the group mean \%PPI conformed the Low-PPI and High-PPI groups, while a Medium-PPI group was randomly drawn from the intermediate values.

The "Pulse" and "No-Pulse" control groups for each strain of the Roman and HS rats underwent sessions of pulse-alone trials (i) or non-stimulus trials (iii), respectively, of the same duration as the PPI session.

Assignment of rats to testing box and time of testing was counterbalanced across groups to avoid order effects. Sample size of each experimental group (Fig. 1), including $n=4-6$ animals in control groups, was determined based on previous c-Fos studies suggesting relatively high homogeneity of c-Fos measures in control groups of similar size (e.g., $[48,49])$.

Tissue processing, c-Fos immunohistochemistry, and microscopy c-Fos immunostaining was performed with modifications as described earlier [50]. Animals were euthanatized $120 \mathrm{~min}$ following the PPI, Pulse and No-Pulse sessions. Brains were removed, immersed in isopentane (SIGMA 320404) and stored at $80^{\circ} \mathrm{C}$ until sectioned. Coronal $20-\mu \mathrm{m}$ thick sections were cut in cryostat (Leica CM3050S) and thaw-mounted onto slides (Thermo scientific). Slides were stored at $-80^{\circ}$. Prior to staining, the slides were allowed to reach RT, then they were immersed in Formaldehyde $3.7-4 \%$ for $12 \mathrm{~min}$ at RT. After fixation, the slices were washed several times in TBS $(0.05,0.15 \mathrm{M}, \mathrm{pH} 7.4)$ and TBS-T $(0.05 \%)$. Endogenous peroxidase activity was quenched with $3 \%$ hydrogen peroxide (SIGMA 216763) in 70\% methanol (SIGMA 179337 ) in $27 \%$ TBS, followed by several washes in TBS-T. Afterwards, endogenous protein was blocked incubating the slices with $10 \%$ normal horse serum (Sigma, H0146) in "antibody diluent" (TBS-T with 1\% (w/v) BSA (SIGMA A9647). Slices were then incubated overnight at $4{ }^{\circ} \mathrm{C}$ with the primary antibody (goat antic-Fos IgG, Santa Cruz, SC52G; 1:250). On the next day, slices were washed in TBS-T, and were then incubated with the secondary antibody (biotinylated horse anti-goat lgG, Vector laboratories, BA9500; 1:200) for $30 \mathrm{~min}$. After washes in TBS-T, the samples were incubated with Peroxidase Streptavidin (HRP conjugated, Jackson immunoresearch, 016-030-084; 1:250) for $30 \mathrm{~min}$. Afterwards, slices were washed in TBS-T, TBS and TB. Then, HRP activity was demonstrated with DAB (50 mg DAB (SIGMA, D5637) $+100 \mathrm{ml}$ of $\mathrm{TB}+33$ microliters of $\mathrm{H}_{2} \mathrm{O}_{2}$ (SIGMA 216763)) for $10 \mathrm{~min}$. Afterwards, slices were washed in TB, dehydrated, cleared, and coverslipped. Microphotographs were captured with an Eclipse 80i Nikon microscope attached to a Nikon DXM1200F70 digital camera at $\times 10$ magnifications. The regions of study were the mPFC (bregma: from 3.72 to $2.52 \mathrm{~mm}$ ); Cg (bregma: from 3.72 to $-1.56 \mathrm{~mm}$ ); dorsal HPC: CA1 of the dorsal HPC (Dorsal CA1), dentate gyrus of the dorsal HPC (Dorsal DG) (bregma: from -2.40 to $-3.84 \mathrm{~mm}$ ); ventral HPC: CA1 of the ventral HPC (Ventral CA1), dentate gyrus of the ventral HPC (Ventral DG) (bregma: from -4.80 to $-5.52 \mathrm{~mm}$ ); NAc: shell and core (bregma: from 2.28 to $1.56 \mathrm{~mm}$ ); dorsal striatum: caudate-putamen (Striatum CP) and globus pallidus (Striatum GP) (bregma: from -1.80 to $-3.12 \mathrm{~mm}$ ); amygdala: basolateral amygdala (BLA) and central amygdala (CeA) (bregma: from -1.92 to $-3.36 \mathrm{~mm}$ ). The borders of each area were identified with the help of a rat brain atlas [51]. See further details of the regions of interest in Fig. S1e-k. The ImageJ software ("analyze particles" function) was employed to automatically identify and count the number of c-Fos immunostained nuclei in three histological sections of each brain region $/ \mathrm{mm}^{2}$ and averaged for each animal. Particle size and appropriate grey threshold were set for each region and maintained for all subjects.

Structural magnetic resonance imaging (MRI)

Settings, parameters and brain region analyses were conducted as previously described [52]. Briefly, we obtained 35 coronal
T2-weighted fast spin-echo images from the olfactory bulb to the cerebellum, which allowed us to manually count pixels of the whole brain, mPFC (bregma: from 3.72 to $2.52 \mathrm{~mm}$ ), Cg (bregma: from 3.72 to $-1.56 \mathrm{~mm}$ ), NAc (including shell and core subregions, bregma: from 2.52 to $0.84 \mathrm{~mm}$ ), HPC (including dorsal and ventral parts, bregma: from -2.40 to $-5.52 \mathrm{~mm}$ ), dorsal striatum (bregma: from 2.28 to $0.96 \mathrm{~mm}$ ), and amygdala (bregma: from -1.20 to $-5.04 \mathrm{~mm}$ ). The borders of each area were identified with the help of a rat brain atlas [51]. See further details of the regions of interest in Fig. S1a-d. Counting of pixels was performed using ImageJ software outlined by an experimenter blinded to group status. Volumes of each delimitated area were calculated using the following formula: [(Field of view $\left(3,5 \times 3,5 \mathrm{~cm}^{2}\right) /$ Matrix size $(256 \times 256)) \times$ Slice thickness $(0.5 \mathrm{~mm})] \times$ number of pixels included in delimitated area. The percentage of relative volume (\% volume) was obtained by using the following formula: [(volume of a delimitated region)/(total brain volume)] $\times 100$. "\% volume" variable was used for analyses in the Roman rat strains, because we found between-strain differences in "total brain volume" measured in $\mathrm{mm}^{3}$. Sample size of each experimental MRI group from studies 2 and 4 (Fig. 1) was determined based on previous MRI studies in rats (e.g., $[27,53])$.

Statistics

All the analyses were performed employing the "Statistics Package for Social Sciences" (SPSS). Significance level was set at $p<0.05$.

A $2 \times 3$ ('2 strains $\times 3$ conditions') ANOVA followed by post hoc Duncan's test were used to determine differences between the RHA and RLA rats in the different conditions (i.e., No-Pulse, Pulse, PPI) of the c-Fos study 1.

Student's $t$-test was used to test significant differences between RHA and RLA rats from study 2.

One-way ANOVA followed by post hoc Duncan's test were used to determine differences among the HS rats with Low-PPI, Medium-PPI and High-PPI, as well as the No-Pulse and Pulse control groups in studies 3 and 4.

To study associations among variables, multiple linear regression (forward stepwise method) and factorial (direct oblimin; oblique rotation) analyses were applied to c-Fos, MRI and PPI data from studies 1,3 , and 4 .

\section{RESULTS}

$\mathrm{PPI}$ and c-Fos in RHA and RLA rats (study 1)

RHA rats had lower PPI than their RLA counterparts (Table S1). No differences were found in "BL1_Startle" between the RHA-Pulse and RLA-Pulse groups, while the RLA-PPI group showed higher baseline startle response (both in BL1 and BL2) than the RHA-PPI group (Table S1). However, analyzing groups of RHA and RLA rats previously matched for their baseline startle response, we found that the between-strain differences in \%PPI were maintained (Fig. S2a, b).

$2 \times 3$ ANOVA revealed a "Strain" effect on c-Fos in the "mPFC" $\left[F_{(1,30)}=6.628 ; p=0.015\right]$ and the $\operatorname{BLA}\left[F_{(1,30)}=7.573 ; p=0.010\right.$; Table 1], as both regions were more activated in RLAs than in their RHA counterparts, and a "Condition" effect in the "mPFC" $\left[F_{(2,30)}=\right.$ $4.488 ; p=0.020]$, the $\mathrm{Cg}\left[F_{(2,30)}=3.488 ; p=0.043\right.$; Table 1] and the CeA $\left[F_{(2,30)}=4.048 ; p=0.028 ;\right.$ Table 1], indicating that these regions had higher activation in the $\mathrm{PPI}$ and Pulse conditions than in the No-Pulse condition in both strains. Importantly, there was a "Strain $\times$ Condition" effect in the $\operatorname{mPFC}\left[F_{(2,30)}=8.243 ; p=0.001\right]$. RLA-PPI rats showed higher c-Fos expression than the RLA-Pulse, RLA-No-Pulse and all RHA groups (Duncan's post hoc test, $p<0.05$ (Fig. 2a); representative microphotographs in Fig. 2b). No significant "Strain" (all $F s_{(1,30)} \leq 4.112$; all $p s \geq 0.052$ ), "Condition" $\left(F s_{(2,30)} \leq 2.358\right.$; all $\left.p s \geq 0.112\right)$ or "Strain $\times$ Condition" $\left(F s_{(2,30)} \leq\right.$ $1.737 ; p s \geq 0.193)$ effects were found in other brain regions (Table 1). 
Table 1. Study 1: c-Fos activation in various brain regions of Roman rats after a single PPI session

\begin{tabular}{|c|c|c|c|c|c|c|c|}
\hline & \multicolumn{7}{|c|}{ Number of c-Fos labeled neurons $/ \mathrm{mm}^{2}$} \\
\hline & No-Pulse $(n=4)$ & Pulse $(n=6)$ & $\mathrm{PPI}(n=8)$ & No-Pulse $(n=4)$ & Pulse $(n=6)$ & $\mathrm{PPI}(n=8)$ & Effects \\
\hline $\mathrm{Cg}$ & $31.4 \pm 1.6$ & $68.6 \pm 10.5^{*}$ & $61.5 \pm 11.2^{*}$ & $33.8 \pm 2.8$ & $52.9 \pm 9.6^{*}$ & $51.6 \pm 9.5^{*}$ & Condition \\
\hline Dorsal CA1 & $3.0 \pm 0.7$ & $3.4 \pm 1.7$ & $3.0 \pm 0.6$ & $3.0 \pm 0.8$ & $4.7 \pm 0.9$ & $5.9 \pm 0.9$ & \\
\hline Dorsal DG & $9.3 \pm 3.0$ & $8.4 \pm 1.2$ & $10.1 \pm 1.3$ & $8.2 \pm 2.8$ & $16.5 \pm 2.9$ & $9.0 \pm 1.3$ & \\
\hline Ventral DG & $4.2 \pm 1.7$ & $7.9 \pm 3.5$ & $11.4 \pm 2.9$ & $6.8 \pm 1.7$ & $11.2 \pm 1.9$ & $7.0 \pm 1.0$ & \\
\hline NAc shell & $23.3 \pm 2.4$ & $28.3 \pm 5.9$ & $35.0 \pm 5.4$ & $21.7 \pm 7.6$ & $38.8 \pm 7.9$ & $24.5 \pm 5.1$ & \\
\hline NAc core & $18.4 \pm 4.3$ & $21.7 \pm 4.6$ & $25.7 \pm 4.6$ & $15.6 \pm 5.8$ & $17.1 \pm 4.6$ & $13.5 \pm 3.0$ & \\
\hline Striatum CP & $3.7 \pm 0.7$ & $10.1 \pm 2.1$ & $7.3 \pm 1.7$ & $7.5 \pm 2.4$ & $9.3 \pm 2.5$ & $11.3 \pm 1.1$ & \\
\hline Striatum GP & $3.3 \pm 1.2$ & $5.4 \pm 0.8$ & $6.7 \pm 1.9$ & $4.9 \pm 3.1$ & $6.4 \pm 2.0$ & $5.5 \pm 1.3$ & \\
\hline
\end{tabular}

$\mathrm{PPI}$ and $\mathrm{MRI}$ in RHA and RLA rats (study 2)

RHA rats had lower PPI than their RLA counterparts (Table S2). No differences were found in "BL1_Startle" and "BL2_Startle" between the RHA and RLA strains (Table S2).

ANOVA revealed a Strain effect in volume (\%) in the mPFC $\left[\mathrm{t}_{(1,18)}=2.189 ; p=0.042\right]$, HPC $\left[\mathrm{t}_{(1,18)}=8.886 ; p=<0.001\right]$ and amygdala $\left[\mathrm{t}_{(1,18)}=5.370 ; p=<0.001\right]$ (Student's $t$-test; Fig. 3a). RHA strain showed lower relative volume of the mPFC, HPC, and amygdala than the RLAs. No statistical differences were found between groups in "\% volume" in other brain regions (all ts $\leq(1,18) 1.737 ; p s \geq 0.193$; Table S3).

PPI and c-Fos in HS rats (study 3)

The expected differences in PPI were found after stratification in the three rat groups, HS Low-PPI $<$ Medium-PPI $<$ High-PPI, while there were no significant differences in "BL1_Startle" and "BL2_Startle" among the three PPI-stratified groups (Table S4). Albeit nonsignificant, a numerical trend towards reduced baseline startle in Low-PPI rats was observed. For this reason, we reanalyzed the data from the HS Low-PPI and High-PPI rats after matching them for their baseline startle response amplitude and found that the betweengroup differences in \%PPI were maintained (Fig. S2c-f).

ANOVA revealed a Group effect in c-Fos expression in the "mPFC" $\left[F_{(4,30)}=7.971 ; p=<0.001\right]$ and "NAc shell" $\left[F_{(4,30)}=2.896\right.$; $p=0.039]$. The number of c-Fos positive cells in the "mPFC" was higher in the High-PPI rats than in the Medium-PPI and Low-PPI groups (Duncan's post hoc test, $p<0.05$; Fig. 2c). Moreover, the High-PPI group was the only one that statistically differed from both the No-Pulse and Pulse control groups. Conversely, in the "NAc shell", the number of c-Fos labeled neurons was greater in the Low-PPI group than in the High-PPI group (Duncan's post hoc test, $p<0.05$; Fig. 2 d). In addition, the Low-PPI group was the only group that statistically differed from the No-Pulse control group. No differences were found among groups in other brain regions (all $F s_{(4,30)} \leq 2.524$; all $p s \geq 0.062$; Table 2a). Multiple regression revealed that $c$-Fos expression in the MPFC and the NAc shell predicted PPI performance $\left(\mathrm{R}^{2}=0.593 ; p=<0.001\right.$; Table S5). In line with that, factor analysis of PPI, startle and c-Fos variables, pooling HS (study 3 ) and Roman rats (study 1), revealed a 6-factor solution, which grouped PPI and MPFC activation (both with positive sign) in the second factor (Table S6).

MRI and c-Fos in HS rats (study 4)

We found the expected differences in \%PPI variables ("PPI 65 70 pre", "PPI total pre", and "PPI 65 post") among the three PPIstratified HS groups: Low-PPI < Medium-PPI < High-PPI. No differences were found in "BL1 Startle pre", "BL2 Startle pre", "BL1_Startle post", and "BL2_Startle post" among the three PPIstratified groups (Table S7).

ANOVA revealed a Group effect in c-Fos expression in the "mPFC" $\left[F_{(4,31)}=4.053 ; p=0.009\right]$, "dorsal CA1" $\left[F_{(4,31)}=2.797 ; p=\right.$ $0.043]$ and "CeA" $\left[F_{(4,31)}=3.624 ; p=0.016\right]$. The Low-PPI group showed lower number of c-Fos positive neurons in the "mPFC" than the Pulse, Medium-PPI and High-PPI groups (Duncan's post hoc test, $p<0.05$; Fig. 2e). In the "dorsal CA1", the High-PPI group also showed higher c-Fos expression than the Low-PPI and NoPulse groups (Duncan's post hoc test, $p<0.05$, Fig. 2f). In the "CeA", the Pulse control group had greater activation than all the PPI groups and the No-Pulse group, while the High-PPI group revealed higher c-Fos expression than the No-Pulse group (Duncan's post hoc test, $p<0.05$, Table 2b). No differences were found among groups in other brain regions (all $F s_{(2,25)} \leq 1.117$; all ps $\geq 0.343$; Table $2 \mathrm{~b}$ ).

Regarding volumetric measures, ANOVA revealed a Group effect in the mPFC $\left[F_{(2,25)}=4.750 ; p=0.018\right]$ and the $\operatorname{HPC}\left[F_{(2,25)}=4.734\right.$; $p=0.018]$. The HS Low-PPI group showed lower MPFC and HPC volumes than the Medium-PPI and High-PPI groups (Duncan's post hoc test, $p<0.05$, Fig. $3 \mathrm{~b}$; see representative MRI images in Fig. 3c, d). No group-related statistical differences were found in other brain regions (all $F s_{(4,31)} \leq 2.552$; all $p s \geq 0.059$; Table S8A). Multiple regression revealed that volumes of the HPC and mPFC predicted PPI levels $\left(\mathrm{R}^{2}=0.513 ; p=0.005\right.$; Table $\left.\mathrm{S} 8 \mathrm{~b}\right)$. In relation to this, factor analysis of study 4 (including PPI, startle, MRI and cFos) showed a 4-factor solution, in which the first factor grouped PPI (pre and post-MRI), mPFC activation and mPFC volume with positive sign (Table S9). 
A) Study 1: c-Fos expression in Roman rats after PPI

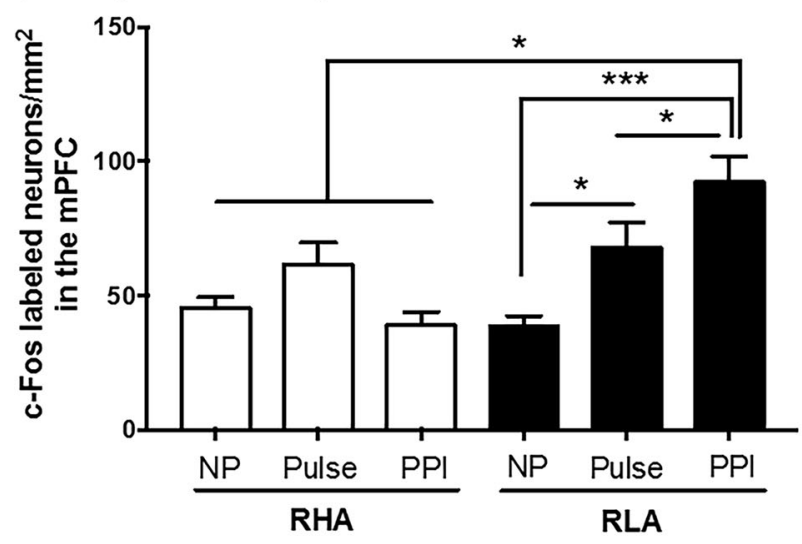

C) Study 3: c-Fos expression in HS rats after PPI

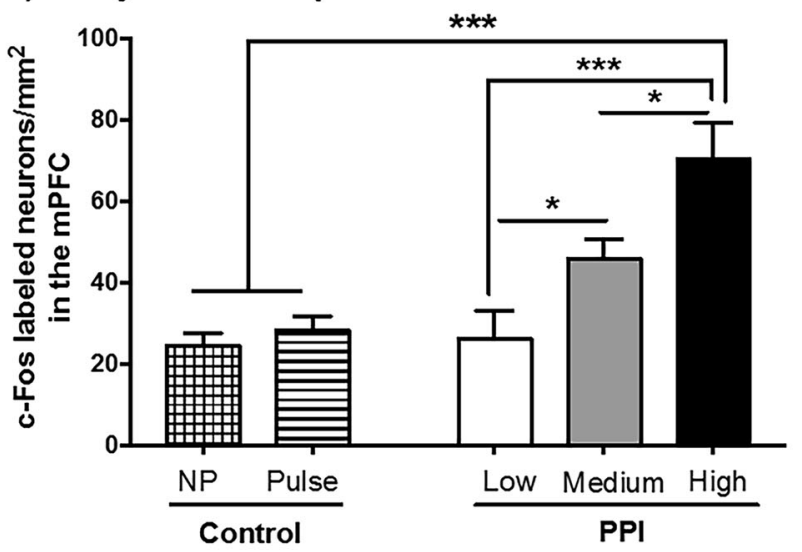

E) Study 4: c-Fos expression in HS rats after 2nd PPI

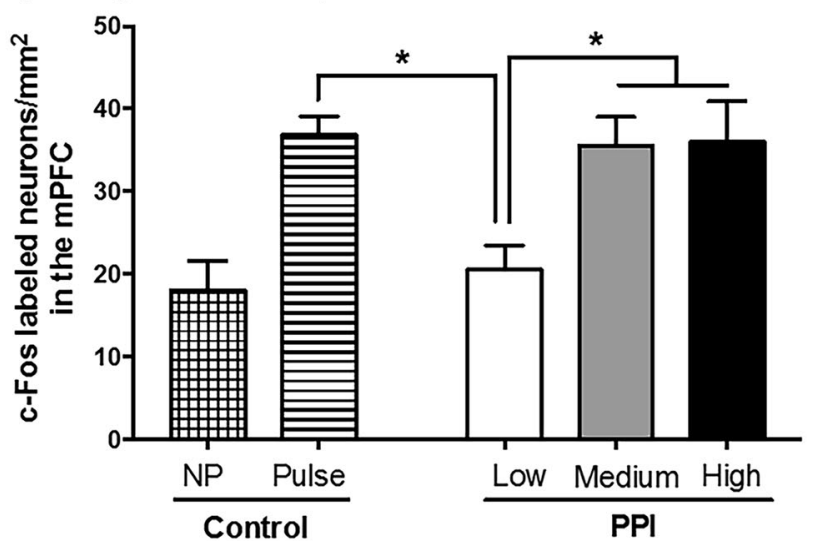

B) Study 1 (mPFC)

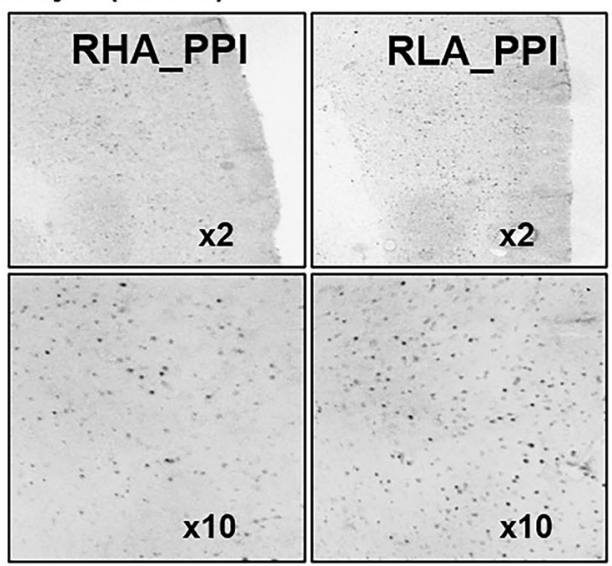

D) Study 3: c-Fos expression in HS rats after PPI

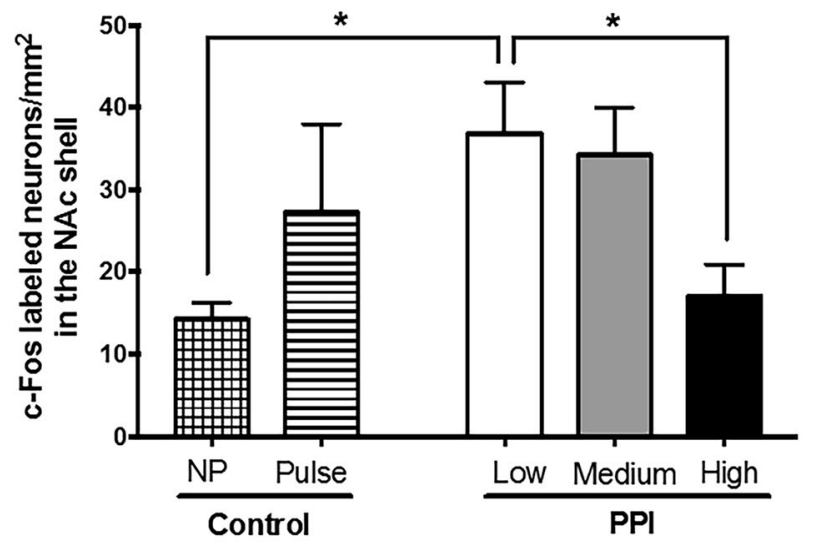

F) Study 4: c-Fos expression in HS rats after 2nd PPI

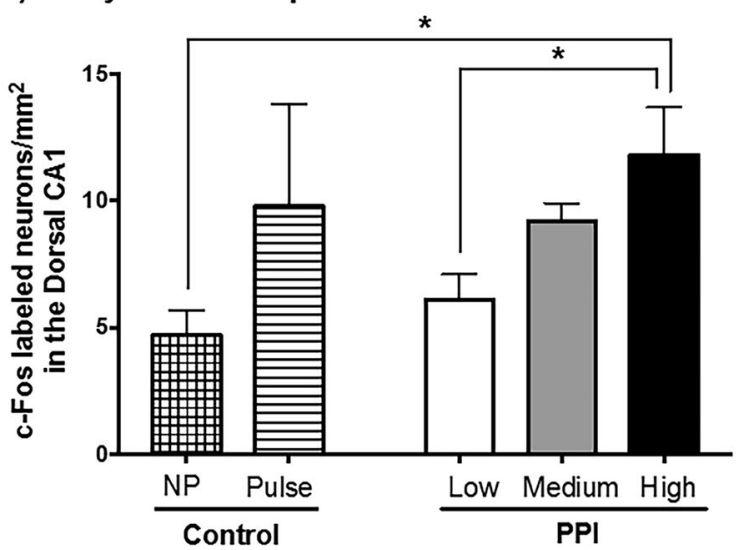

Fig. 2 Reduced PPI is associated with decreased medial prefrontal cortex (mPFC) activation in Roman and HS rats. a mPFC activation in RLA rats was significantly higher in the PPI condition than in the NP (No-Pulse) and Pulse conditions and was also higher than all conditions in the RHA rats. Conversely, RHA rats did not show differences in mPFC activation among the three conditions. b Representative photomicrographs of c-Fos differences in the mPFC (c-Fos expression) between the Roman rats. RHA rats exposed to a PPI session (RHA PPI) had lower mPFC activation than their RLA (RLA_PPI) counterparts, as observed both at $\times 2$ and $\times 10$ magnifications. $c$ HS rats stratified for high-PPI scores (HighPPI) showed increased neuronal activity in the mPFC compared to HS rats stratified by medium-PPI scores (Medium-PPI) and HS rats stratified by low-PPI scores (Low-PPI). The Medium-PPI had higher mPFC activation than the Low-PPI. Additionally, the High-PPI group showed higher mPFC activation than the No-pulse control group (NP, control) and the Pulse control group (Pulse, control). $\mathbf{d}$ Nucleus accumbens shell (NAc shell) activation in the Low-PPI group was greater than in the High-PPI and NP groups. e Following a second PPI session (study 4), the HS LowPPI group showed lower c-Fos expression in the mPFC than Medium-PPI, High-PPI and Pulse groups. $f$ The dorsal CA1 of the hippocampus (Dorsal CA1) in the the High-PPI group had higher c-Fos expression than the Low-PPI and NP groups. Values are mean \pm SEM. See " $n$ "/group in Fig. $1 .{ }^{*} p<0.05,{ }^{* *} p<0.01,{ }^{* * *} p<0.001$ (Duncan's multiple range test) 
A) Study 2: MRI in Roman rats

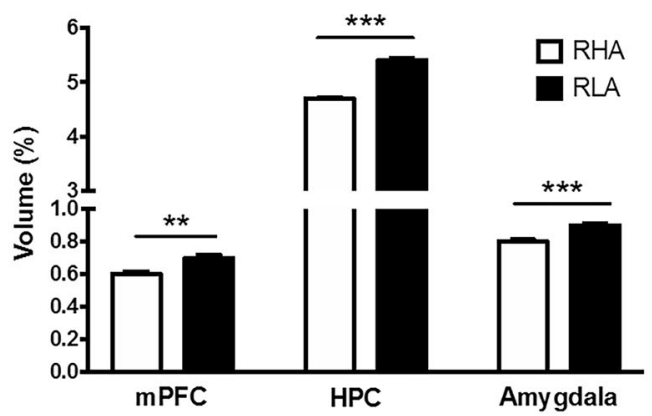

C) Study 4 (MPFC)

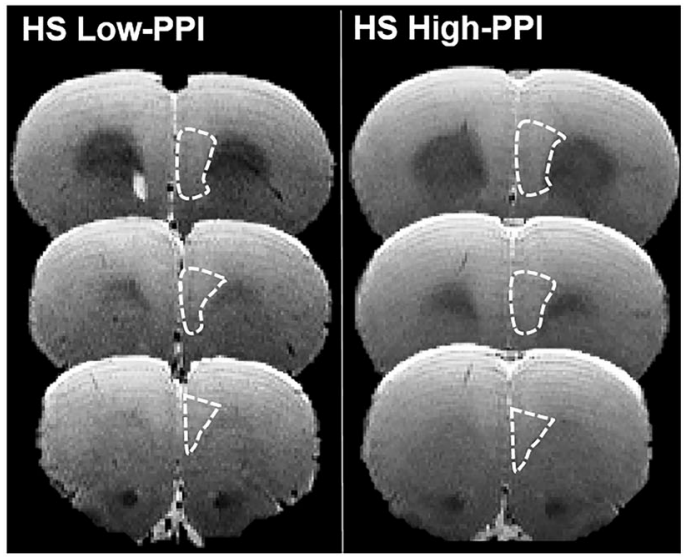

B) Study 4: MRI in HS rats

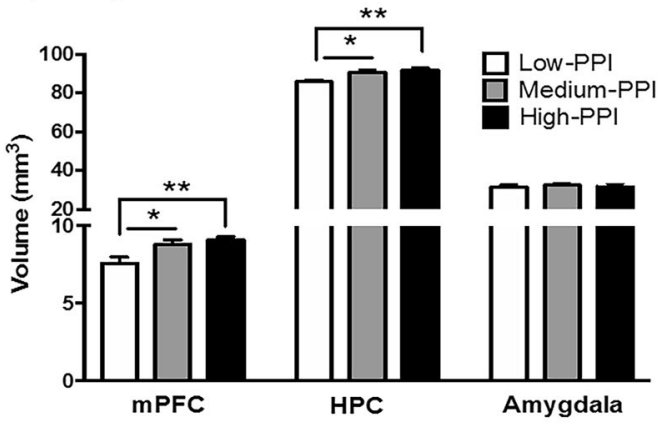

D) Study 4 (HPC)

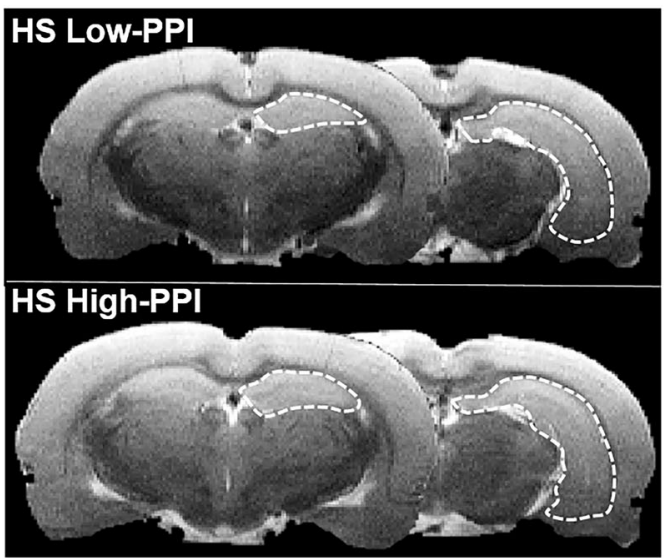

Fig. 3 Lower PPI is associated with decreased MRI volumes in the medial prefrontal cortex (mPFC) and hippocampus (HPC) in Roman and HS rats. a RHA rats showed significant reductions in the "\% volume" of the MPFC, HPC and amygdala compared to their RLA counterparts. "\% volume" variable was used for analyses in the Roman rat strains, because we found between-strain differences in "total brain volume" measured in $\mathrm{mm}^{3}$ (see Table S3). b The HS rats stratified by low PPI (Low-PPI) had lower mPFC and HPC volumes than HS rats stratified by medium (Medium-PPI) or high (High-PPI) PPI. No statistical differences were found in the amygdala. Values are mean $\pm \mathrm{SEM} .{ }^{*} p<0.05,{ }^{* *} p<$ $0.01,{ }^{* *} p<0.001$ (Student's $t$-test in "a"; Duncan's multiple range test in "b"). c, d Representative coronal MRI images showing volumetric differences in the MPFC (c) and HPC (d) between the HS Low-PPI and High-PPI rats. The HS Low-PPI showed a thinner mPFC and HPC (white dashed lines) than HS High-PPI rats. The borders of each area were identified with the help of a rat brain atlas [51]

\section{DISCUSSION}

This work was aimed to investigate the neurobiological mechanisms associated with reduced sensorimotor gating in the Roman and in the outbred heterogeneous HS rats. Our results showed that both neuronal activity and volume of the MPFC, as well as HPC volume, were highly and consistently associated with spontaneous differences in PPI in intact rats.

PPI is related to MPFC activation and MPFC and HPC volumes in Roman and HS rats

Results of c-Fos expression in the Roman rats (study 1 ) indicated that the RLA rats showed higher activation of the MPFC than the RHAs during the PPI test. Interestingly, the different conditions markedly affected RLA rats, i.e., much higher c-Fos activation in the PPI condition than the No-Pulse and Pulse conditions, while did not significantly influence c-Fos expression in RHA rats. Moreover, c-Fos results from study 3 revealed that the HS High-PPI group had higher neuronal activity in the MPFC than all the other four groups, thus suggesting specificity of the mPFCPPI association. Similarly, c-Fos results in HS rats from study 4 showed that the Low-PPI group displayed lower mPFC activation than the High-PPI, Medium-PPI and Pulse groups. The differences in c-Fos expression between both studies using $\mathrm{HS}$ rats (Fig. 2c, e) may be due to habituation, since all animals from study 4 were tested twice in the PPI apparatus. Importantly, it is noteworthy that differences in c-Fos activation between the Pulse and No-Pulse control groups and the PPI groups in study 4 may reflect PPI-related mechanism, although it cannot be excluded that these differences reflect divergences in the experimental history between control and PPI groups, including that PPI groups had undergone MRI measurements under anesthesia.

The main conclusion arising from studies 1,3 , and 4 is that there is a strong and consistent positive relationship between activation of the MPFC and PPI response, which is generalizable through different genetic backgrounds (i.e., different rat strains). Remarkably, this is globally supported by regression analysis revealing that activation of the mPFC (together with NAc shell) predicts PPI levels, and by factor analysis (pool of studies 1 and 3, Table S6). In contrast, the $\mathrm{Cg}$, which is a region adjacent to the $\mathrm{MPFC}$ and that has been previously related to schizophrenia ([54] but see also [55]), was equally activated by PPI and Pulse conditions in study 1 in both RHA and RLA and there were no differences among HS rats (study 3). This result reinforces the specificity of the association between mPFC activation and PPI. Importantly, our findings agree with previous studies showing that PPI is: (i) positively associated with $\mathrm{mPFC}$ activation in rats selectively bred for low or high PPI [56]; (ii) impaired after several pharmacological and neurodevelopmental treatments in the $\operatorname{mPFC}[9-11,24,25]$; or (iii) improved in the poly I:C rat model treated with electrical stimulation of the mPFC $[28,29]$. However, in the only previous study using untreated and unselected animals, there was no difference in c-Fos expression between PPI-exposed and Pulseexposed mice in the MPFC [48]. One possible explanation for the 
Table 2. c-Fos activation in various brain regions in HS rats from studies 3 and 4

(A) Study 3: c-Fos activation in various brain regions of HS rats after PPI

\begin{tabular}{|c|c|c|c|c|c|}
\hline & \multicolumn{5}{|c|}{ Number of c-Fos labeled neurons $/ \mathrm{mm}^{2}$} \\
\hline $\mathrm{Cg}$ & $53.2 \pm 5.4$ & $58.6 \pm 18.2$ & $66.9 \pm 9.8$ & $75.2 \pm 14.3$ & $84.8 \pm 13.0$ \\
\hline Dorsal DG & $11.4 \pm 2.3$ & $10.0 \pm 3.1$ & $11.6 \pm 2.6$ & $10.2 \pm 1.0$ & $5.0 \pm 0.7$ \\
\hline Ventral CA1 & $11.2 \pm 0.8$ & $11.6 \pm 2.1$ & $12.2 \pm 1.6$ & $14.2 \pm 1.3$ & $12.6 \pm 2.6$ \\
\hline Striatum CP & $9.5 \pm 3.5$ & $13.7 \pm 4.5$ & $13.7 \pm 3.3$ & $12.4 \pm 2.4$ & $15.6 \pm 2.5$ \\
\hline Striatum GP & $5.1 \pm 1.5$ & $8.1 \pm 3.7$ & $8.0 \pm 1.3$ & $6.3 \pm 1.5$ & $6.3 \pm 1.2$ \\
\hline BLA & $10.5 \pm 4.1$ & $8.4 \pm 2.6$ & $13.7 \pm 2.3$ & $19.7 \pm 2.0$ & $15.8 \pm 3.0$ \\
\hline $\mathrm{CeA}$ & $7.9 \pm 1.8$ & $12.3 \pm 4.3$ & $15.4 \pm 5.5$ & $10.1 \pm 1.4$ & $8.0 \pm 1.5$ \\
\hline
\end{tabular}

(B) Study 4: c-Fos activation in various brain regions of $\mathrm{HS}$ rats after second PPI

\begin{tabular}{|c|c|c|c|c|c|}
\hline & $(n=4)$ & $(n=4)$ & $(n=7)$ & $(n=12)$ & $(n=9)$ \\
\hline Dorsal DG & $25.1 \pm 7.1$ & $22.6 \pm 2.8$ & $25.4 \pm 4.0$ & $24.5 \pm 3.0$ & $24.8 \pm 3.3$ \\
\hline Ventral DG & $9.3 \pm 1.0$ & $8.6 \pm 2.1$ & $16.0 \pm 1.7$ & $16.4 \pm 2.1$ & $12.3 \pm 1.6$ \\
\hline NAc shell & $13.5 \pm 2.3$ & $17.9 \pm 9.7$ & $21.3 \pm 5.4$ & $15.0 \pm 3.0$ & $20.8 \pm 3.3$ \\
\hline NAc core & $9.8 \pm 2.7$ & $11.9 \pm 4.2$ & $11.2 \pm 1.8$ & $14.9 \pm 1.6$ & $12.6 \pm 1.9$ \\
\hline Striatum GP & $6.3 \pm 1.6$ & $8.1 \pm 1.7$ & $11.6 \pm 1.8$ & $9.0 \pm 1.6$ & $10.9 \pm 1.6$ \\
\hline BLA & $17.5 \pm 7.2$ & $26.8 \pm 3.6$ & $20.7 \pm 3.4$ & $20.1 \pm 2.3$ & $23.9 \pm 3.1$ \\
\hline $\mathrm{CeA}$ & $8.4 \pm 3.2^{*}$ & $26.8 \pm 3.2$ & $17.2 \pm 2.2^{*}$ & $14.7 \pm 1.6^{*}$ & $18.0 \pm 3.4^{* \#}$ \\
\hline
\end{tabular}

(A) In study 3, HS rats were stratified by their PPI scores in rats with low-PPI scores (Low-PPI), medium-PPI scores (Medium-PPI) and high-PPI scores (High-PPI). Moreover, control groups for background noise (No-Pulse) and pulse-alone (Pulse) were used. (B) In study 4, one week after undergoing an MRI session, HS rats were submitted to another PPI session to obtain samples to conduct c-Fos analyses. See further procedural details in "Materials and Methods" and Fig. 1. Values are mean \pm SEM. Abbreviations as in Table 1.

${ }^{*} p<0.05$ vs. Pulse group, ${ }^{\#} p<0.05$ vs. No-Pulse group in the CeA (Duncan's multiple range test following significant ANOVA effect)

discrepancy with our findings is that in the mice study, there was just a PPI-exposed group, and animals were not stratified by differential PPI. In fact, the average percentage of PPI in that study was around $50 \%$, which roughly corresponds to Low-PPI rats in the present study 3. Moreover, it is not yet clear that the same neural mechanisms are involved in the regulation of PPI in different species (e.g., mice and rats) $[13,48]$.

Conversely, we found no group-related differences in c-Fos expression in the different HPC sub-regions in studies 1 and 3 . These results may seem surprising in light of the literature relating HPC function and sensorimotor gating [5, 13, 32], although there have also been some controversial findings $[48,57]$. However, in study 4 the Dorsal CA1 had higher activation in the HS High-PPI group than in the Low-PPI and No-Pulse groups. In this regard, it is assumed that the Dorsal CA1 is critically involved in several types of memory [58]. As animals were tested twice for PPI in study 4, this may suggest that such an increase of c-Fos activation in the Dorsal CA1 of High-PPI rats is due to a memory process (i.e., habituation of PPI).

In contrast to our findings, previous studies show that PPI is also impaired by manipulations that increase activity in the mPFC [12] or the HPC [14-18]. However, recent evidence suggests that the hippocampal and prefrontal modulation of PPI may be strain-dependent as, for instance, prefrontal disinhibition in Sprague-Dawley rats [12] and ventral hippocampal disinhibition in Wistar rats [16] disrupt PPI, but none of these effects are observed in Lister hooded rats $[59,60]$.

Regarding the MRI results, study 2 revealed that RHA rats had lower "\% volume" of the mPFC and the HPC than the RLAs. These findings agree with previous evidence showing decreased volume of both areas and reduced PPI in RHA compared with RLA rats, as well as a significant positive correlation between HPC volume and PPI $[52,61]$. In keeping with that, in the present study 4, the HS LowPPI group had lower mPFC and HPC volumes than the Medium-PPI and High-PPI groups. Regression analysis supports these findings, as mPFC and HPC volumes positively predict PPI levels (see Table S8b). In this regard, it is known that variations in the volume of particular brain regions may reflect microscopic changes within these regions, including changes in synaptogenesis, dendritic arborization, number of neurites, and neuronal and glial genesis, that can, in turn, influence behavioral responses [62-64]. For instance, a study using a "double hit" rat model of schizophrenia reports volume reductions in the mPFC accompanied by several neurochemical alterations in cortical inhibitory circuits within this region, such as a reduction in parvalbumin expressing interneurons, in mRNA levels of calbindin and ERbB4, and in expression of PSA-NCAM and GAD67 [55]. To the extent that macroscopic variations in the volume of a specific brain region are accompanied by microscopic changes that can influence behavioral processes, our MRI results suggest that the MPFC and HPC may regulate PPI. Moreover, our 
experimental strategy (study 4) enabled us to study PPI, MRI and cFos in the same subjects. Thus, factor analysis of the most relevant parameters (explaining $64.1 \%$ variance) revealed a 4 -factor solution, with a first factor grouping PPI variables with mPFC volume and activation (see Table S9), which is globally consistent with the results of group comparisons and regression analyses (studies 3-4). Our findings are in line with previous reports showing both impaired PPI and reduced MPFC volume in several neurodevelopmental rat models $[10,26,27,53]$. In addition, MRI studies in schizophrenic patients have shown significant correlations between PPI and gray matter volume in the dorsolateral prefrontal, middle frontal and orbital/medial prefrontal cortices [8], whereas HPC volume correlated with PPI in healthy subjects [33]. Moreover, patients with first-episode schizophrenia show significant volumetric reductions in the HPC [65].

The present strategy, which considers the spontaneous variation of PPI in intact rats, may be a parallel of the study of brain function/structure and PPI in humans. Nevertheless, the rat model approach has the obvious advantage of enabling application of invasive procedures and enhanced control of variables. For example, our c-Fos results are in line with a previous functional MRI (fMRI) study in humans that demonstrated that patients with schizophrenia had lower activation of the frontal and parietal cortical regions than healthy subjects during a PPI test [32]. Importantly, however, the higher spatial resolution of c-Fos over fMRI improves detection of very small nuclei and single neurons involved, which is not applicable to humans $[66,67]$.

NAc and amygdala activation during PPI in the Roman and HS rats The HS Low-PPI group showed higher NAc shell activity than both the High-PPI group and the No-Pulse control group (study 3), revealing an opposite relationship with PPI compared to the findings from the mPFC (see also regression analysis in Table S5). A similar, albeit non-significant, trend was observed in study 1 between the Roman strains, as the mean c-Fos value of the PPIdeficient RHA rats was $35.0( \pm 5.4)$ vs. $24.5( \pm 5.1)$ of their RLA counterparts (see Table 1). These findings are consistent with evidence of reduced neuronal activity in the MPFC and increased activity in the NAc of rats selectively bred for deficient sensorimotor gating [56]. Nevertheless, it is worth to point out that the relationship between NAc function and PPI is still a matter of controversy. In fact, PPI is impaired after (i) ablative lesion of the NAc shell [19]; (ii) functional inhibition of the NAc core, but not shell [23]; (iii) amphetamine infusion into the NAc core, but not the NAc shell [68]; or (iv) high-frequency electrical stimulation of the NAc shell in control-saline offspring, while somewhat paradoxically it alleviates PPI deficits in the poly I:C offspring [29]. On the other hand, it has been reported that direct stimulation of alfa-1 and beta adrenoceptors in the NAc shell, but not core, disrupts $\mathrm{PPI}$, and that blocking alfa-1 receptors within NAc shell reverses amphetamine-induced PPI impairments [69]. In relation to this, the acute administration of methylphenidate, which stimulates dopamine transmission in the NAc, elicits an increase in c-Fos expression in both the NAc shell and core, but more markedly in the former, in parallel to PPI impairments in mice [70]. In addition, the disruptive effects of PPI by dopamine agonists seem to be strain-dependent, as Sprague-Dawley are more sensitive to PPIdisruptive effects of apomorphine and amphetamine than Lister Hooded rats $[5,68]$. To sum up, studies investigating NAc function and PPI have provided mixed and even paradoxical results, which indicates the need for further research. Our results, however, would be in line with those findings suggesting that a hyperactive NAc shell is associated with reduced PPI. Nevertheless, in study 4 no trend for a negative association between NAc shell activation and PPI was observed, which is likely because c-Fos expression was measured after repeated PPI exposure. In this context, there is wide evidence that repeated exposure to a novel environment or stress leads to decreased c-Fos response to those stimuli [71].
Therefore, c-Fos results from study 4 may be to some extent influenced by a process of habituation [72].

On the other hand, previous studies suggest that both the amygdala and NAC may interact to modulate PPI [2]. However, we did not find a specific relationship between PPI and activation in the CeA or BLA. Instead, there was (i) an unspecific "pulse" effect in the $\mathrm{CeA}$, as reflected by higher c-Fos activation in the PPI and Pulse conditions than in the No-Pulse condition (studies 1 and 4); and (ii) a "strain" effect in the BLA in study 1, as the BLA was globally more activated in RLAs than in their RHA counterparts. This finding, together with the increased amygdala volume in RLA rats (see Fig. 3a), is consistent with previous results of strainrelated differential stress sensitivity and amygdala volume and function $[49,52]$.

\section{CONCLUSIONS}

PPI is a translational measure of sensorimotor gating that is impaired in several human diseases, such as schizophrenia, and is used in many rodent animal models to investigate brain mechanisms underlying these diseases. A common approach in rodent studies has been to assess the impact of specific brain manipulations on PPI, while neural correlates of PPI in humans have preferentially been studied using brain imaging. In this regard, our present work might contribute to bridge the gap between rodent and human studies by investigating structural and functional brain correlates in intact inbred and outbred rats with spontaneous differences in PPI. We report a consistent positive association between PPI and MPFC activity and volume. Moreover, our results suggest that, apart from a hypoactive and smaller mPFC, a hyperactive NAc shell and a smaller HPC may underlie reduced PPI levels. Overall, our findings support the notion that sensorimotor gating is modulated by forebrain structures and highlight the importance of the mPFC in its regulation.

\section{FUNDING AND DISCLOSURE}

This work was supported by the following grants: PSI2017-82257-P (MINECO), 2017SGR-1586, and "ICREA-Academia 2013" [to AF-T] and by the following Ph.D. fellowships: FPU (FPU15/06307 [to CTE]), FPI [to AS-G] and FI [to IO]. The authors declare no competing interests.

\section{ADDITIONAL INFORMATION}

Supplementary Information accompanies this paper at (https://doi.org/10.1038/ s41386-019-0392-x).

Publisher's note: Springer Nature remains neutral with regard to jurisdictional claims in published maps and institutional affiliations.

\section{REFERENCES}

1. Graham FK. The more or less startling effects of weak prestimulation. Psychophysiology. 1975;12:238-48.

2. Kohl S, Heekeren K, Klosterkötter J, Kuhn J. Prepulse inhibition in psychiatric disorders-apart from schizophrenia. J Psychiatr Res. 2013:47:445-52.

3. Braff D, Stone C, Callaway E, Geyer M, Glick I, Bali L. Prestimulus effects on human startle reflex in normals and schizophrenics. Psychophysiology. 1978;15:339-43.

4. Swerdlow NR, Light GA, Sprock J, Calkins ME, Green MF, Greenwood TA, et al. Deficient prepulse inhibition in schizophrenia detected by the multi-site COGS. Schizophr Res. 2014;152:503-12.

5. Swerdlow NR, Geyer MA, Braff DL. Neural circuit regulation of prepulse inhibition of startle in the rat: current knowledge and future challenges. Psychopharmacol (Berl). 2001;156:194-215.

6. Swerdlow NR, Geyer MA. Using an animal model of deficient sensorimotor gating to study the pathophysiology and new treatments of schizophrenia. Schizophr Bull. 1998;24:285-301. 
7. Geyer MA, Krebs-Thomson K, Braff DL, Swerdlow NR. Pharmacological studies of prepulse inhibition models of sensorimotor gating deficits in schizophrenia: a decade in review. Psychopharmacol (Berl). 2001;156:117-54.

8. Kumari V, Fannon D, Geyer MA, Premkumar P, Antonova E, Simmons A, et al. Cortical grey matter volume and sensorimotor gating in schizophrenia. Cortex. 2008:44:1206-14.

9. Koch M, Bubser M. Deficient sensorimotor gating after 6-hydroxydopamine lesion of the rat medial prefrontal cortex is reversed by haloperidol. Eur J Neurosci. 1994;6:1837-45.

10. Schneider $M$, Koch M. Behavioral and morphological alterations following neonatal excitotoxic lesions of the medial prefrontal cortex in rats. Exp Neurol. 2005;195:185-98.

11. Uehara T, Sumiyoshi T, Matsuoka T, Itoh H, Kurachi M. Effect of prefrontal cortex inactivation on behavioral and neurochemical abnormalities in rats with excitotoxic lesions of the entorhinal cortex. Synapse. 2007;61:391-400.

12. Japha K, Koch M. Picrotoxin in the medial prefrontal cortex impairs sensorimotor gating in rats: reversal by haloperidol. Psychopharmacol (Berl). 1999;144:347-54.

13. Swerdlow NR, Light GA. Animal models of deficient sensorimotor gating in schizophrenia: are they still relevant? Curr Top Behav Neurosci. 2016;28:305-25.

14. Howland JG, MacKenzie EM, Yim TT, Taepavarapruk P, Phillips AG. Electrical stimulation of the hippocampus disrupts prepulse inhibition in rats: frequency- and site-dependent effects. Behav Brain Res. 2004;152:187-97.

15. Bast T, Zhang WN, Heidbreder C, Feldon J. Hyperactivity and disruption of prepulse inhibition induced by $\mathrm{N}$-methyl-D-aspartate stimulation of the ventral hippocampus and the effects of pretreatment with haloperidol and clozapine. Neuroscience. 2001;103:325-35.

16. Bast T, Zhang WN, Feldon J. Hyperactivity, decreased startle reactivity, and disrupted prepulse inhibition following disinhibition of the rat ventral hippocampus by the $\operatorname{GABA}(\mathrm{A})$ receptor antagonist picrotoxin. Psychopharmacol (Berl). 2001;156:225-33.

17. Zhang W-N, Bast T, Feldon J. Effects of hippocampal N-methyl-D-aspartate infusion on locomotor activity and prepulse inhibition: differences between the dorsal and ventral hippocampus. Behav Neurosci. 2002;116:72-84.

18. Nguyen R, Morrissey MD, Mahadevan V, Cajanding JD, Woodin MA, Yeomans JS, et al. Parvalbumin and GAD65 interneuron inhibition in the ventral hippocampus induces distinct behavioral deficits relevant to schizophrenia. J Neurosci. 2014;34:14948-60.

19. Kodsi MH, Swerdlow NR. Reduced prepulse inhibition after electrolytic lesions of nucleus accumbens subregions in the rat. Brain Res. 1997;773:45-52.

20. Wan FJ, Geyer MA, Swerdlow NR. Accumbens D2modulation of sensorimotor gating in rats: assessing anatomical localization. Pharm Biochem Behav. 1994:49:155-63.

21. Wan FJ, Geyer MA, Swerdlow NR. Presynaptic dopamine-glutamate interactions in the nucleus accumbens regulate sensorimotor gating. Psychopharmacol (Berl). 1995;120:433-41.

22. Wan FJ, Swerdlow NR. Sensorimotor gating in rats is regulated by different dopamine-glutamate interactions in the nucleus accumbens core and shell subregions. Brain Res. 1996;722:168-76.

23. Pothuizen $\mathrm{HHJ}$, Jongen-Rêlo AL, Feldon J. The effects of temporary inactivation of the core and the shell subregions of the nucleus accumbens on prepulse inhibition of the acoustic startle reflex and activity in rats. Neuropsychopharmacology. 2005;30:683-96.

24. Toriumi K, Oki M, Muto E, Tanaka J, Mouri A, Mamiya T, et al. Prenatal phencyclidine treatment induces behavioral deficits through impairment of GABAergic interneurons in the prefrontal cortex. Psychopharmacol (Berl). 2016;233:2373-81.

25. Wischhof L, Irrsack E, Osorio C, Koch M. Prenatal LPS-exposure - a neurodevelopmental rat model of schizophrenia-differentially affects cognitive functions, myelination and parvalbumin expression in male and female offspring. Prog Neuropsychopharmacol Biol Psychiatry. 2015;57:17-30.

26. Day-Wilson KM, Jones DNC, Southam E, Cilia J, Totterdell S. Medial prefrontal cortex volume loss in rats with isolation rearing-induced deficits in prepulse inhibition of acoustic startle. Neuroscience. 2006;141:1113-21.

27. Schubert MI, Porkess MV, Dashdorj N, Fone KCF, Auer DP. Effects of social isolation rearing on the limbic brain: a combined behavioral and magnetic resonance imaging volumetry study in rats. Neuroscience. 2009;159:21-30.

28. Klein J, Hadar R, Götz T, Männer A, Eberhardt C, Baldassarri J, et al. Mapping brain regions in which deep brain stimulation affects schizophrenia-like behavior in two rat models of schizophrenia. Brain Stimul. 2013;6:490-9.

29. Bikovsky L, Hadar R, Soto-Montenegro ML, Klein J, Weiner I, Desco M, et al. Deep brain stimulation improves behavior and modulates neural circuits in a rodent model of schizophrenia. Exp Neurol. 2016;283:142-50.

30. Hazlett EA, Buchsbaum MS. Sensorimotor gating deficits and hypofrontality in schizophrenia. Front Biosci. 2001;6:D1069-72.

31. Sawa A, Snyder SH. Schizophrenia: diverse approaches to a complex disease. Science (80-). 2002;296:692-5.
32. Kumari V, Gray JA, Geyer MA, Ffytche D, Soni W, Mitterschiffthaler MT, et al. Neural correlates of tactile prepulse inhibition: a functional MRI study in normal and schizophrenic subjects. Psychiatry Res. 2003;122:99-113.

33. Kumari V, Antonova E, Zachariah E, Galea A, Aasen I, Ettinger U, et al. Structural brain correlates of prepulse inhibition of the acoustic startle response in healthy humans. Neuroimage. 2005;26:1052-8.

34. Escorihuela RM, Fernández-Teruel A, Gil L, Aguilar R, Tobeña A, Driscoll P. Inbred roman high- and low-avoidance rats: differences in anxiety, novelty-seeking, and shuttlebox behaviors. Physiol Behav. 1999;67:19-26.

35. Río-Alamos C, Oliveras I, Cañete T, Blázquez G, Martínez-Membrives E, Tobeña A, et al. Neonatal handling decreases unconditioned anxiety, conditioned fear, and improves two-way avoidance acquisition: a study with the inbred Roman high (RHA-I)- and low-avoidance (RLA-I) rats of both sexes. Front Behav Neurosci. 2015;9:174

36. Oliveras I, Río-Álamos C, Cañete T, Blázquez G, Martínez-Membrives E, Giorgi O, et al. Prepulse inhibition predicts spatial working memory performance in the inbred Roman high- and low-avoidance rats and in genetically heterogeneous $\mathrm{NIH}-\mathrm{HS}$ rats: relevance for studying pre-attentive and cognitive anomalies in schizophrenia. Front Behav Neurosci. 2015;9:213.

37. Esnal A, Sánchez-González A, Río-Álamos C, Oliveras I, Cañete T, Blázquez G, et al Prepulse inhibition and latent inhibition deficits in Roman high-avoidance vs. Roman low-avoidance rats: modeling schizophrenia-related features. Physiol Behav. 2016;163:267-73

38. Giorgi O, Piras G, Corda MG. The psychogenetically selected Roman high- and low-avoidance rat lines: a model to study the individual vulnerability to drug addiction. Neurosci Biobehav Rev. 2007:31:148-63.

39. Tournier BB, Steimer T, Millet $P$, Moulin-Sallanon $M$, Vallet $P$, Ibañez $V$, et al Innately low D2 receptor availability is associated with high novelty-seeking and enhanced behavioural sensitization to amphetamine. Int J Neuropsychopharmacol. 2013;16:1819-34.

40. Klein $A B$, Ultved $L$, Adamsen $D$, Santini MA, Tobeña A, Fernandez-Teruel $A$, et al $5-\mathrm{HT}(2 \mathrm{~A})$ and mGlu2 receptor binding levels are related to differences in impulsive behavior in the Roman Low- (RLA) and High- (RHA) avoidance rat strains. Neuroscience. 2014;263:36-45.

41. Wood CM, Nicolas CS, Choi SL, Roman E, Nylander I, Fernandez-Teruel A, et al. Prevalence and influence ofcys $407^{*} \mathrm{Grm} 2$ mutation in Hannover-derived Wistar rats: mGlu2 receptor loss links to alcohol intake, risk taking and emotional behaviour. Neuropharmacology. 2017;115:128-38.

42. Fomsgaard L, Moreno $\mathrm{L}$, de la Fuente Revenga M, Brudek T, Adamsen D, RioAlamos $C$, et al. Differences in 5-HT2A and mGlu2 receptor expression levels and repressive epigenetic modifications at the $5-\mathrm{HT} 2 \mathrm{~A}$ promoter region in the Roman Low- (RLA-I) and High- (RHA-I) Avoidance Rat Strains. Mol Neurobiol. 2018;55:1998-2012.

43. Hansen C, Spuhler K. Development of the National Institutes of Health Genetically Heterogeneous Rat Stock. Alcohol Clin Exp Res. 1984;8:477-9.

44. Baud A, Hermsen R, Guryev V, Stridh P, Graham D, McBride MW, et al. Combined sequence-based and genetic mapping analysis of complex traits in outbred rats. Nat Genet. 2013;45:767-75.

45. Díaz-Morán S, Palència $M$, Mont-Cardona $C$, Cañete $T$, Blázquez G, MartínezMembrives $\mathrm{E}_{\text {, }}$ et al. Gene expression in hippocampus as a function of differential trait anxiety levels in genetically heterogeneous NIH-HS rats. Behav Brain Res. 2013;257:129-39.

46. Sánchez-González A, Esnal A, Río-Álamos C, Oliveras I, Cañete T, Blázquez G, et al. Association between prepulse inhibition of the startle response and latent inhibition of two-way avoidance acquisition: a study with heterogeneous NIH-HS rats. Physiol Behav. 2016;155:195-201.

47. Tapias-Espinosa C, Río-Álamos C, Sampedro-Viana D, Gerbolés C, Oliveras I, Sánchez-González A, et al. Increased exploratory activity in rats with deficient sensorimotor gating: a study of schizophrenia-relevant symptoms with genetically heterogeneous NIH-HS and Roman rat strains. Behav Process. 2018;151:96-103.

48. Takahashi $K$, Nagai T, Kamei $H$, Maeda K, Matsuya T, Arai $S$, et al. Neural circuits containing pallidotegmental GABAergic neurons are involved in the prepulse inhibition of the startle reflex in mice. Biol Psychiatry. 2007;62:148-57.

49. Meyza KZ, Boguszewski PM, Nikolaev E, Zagrodzka J. Diverse sensitivity of RHA/ Verh and RLA/Verh rats to emotional and spatial aspects of a novel environment as a result of a distinct pattern of neuronal activation in the fear/anxiety circuit. Behav Genet. 2009;39:48-61.

50. Sundquist SJ, Nisenbaum LK. Fast Fos: rapid protocols for single- and doublelabeling c-Fos immunohistochemistry in fresh frozen brain sections. J Neurosci Methods. 2005;141:9-20.

51. Paxinos G, Watson C. The Rat Brain in Stereotaxic. The Nether. The Netherlands: Elsevier; 2007.

52. Río-Álamos C, Oliveras I, Piludu MA, Gerbolés C, Cañete T, Blázquez G, et al. Neonatal handling enduringly decreases anxiety and stress responses and 
reduces hippocampus and amygdala volume in a genetic model of differential anxiety: behavioral-volumetric associations in the Roman rat strains. Eur Neuropsychopharmacol. 2017;27:146-58.

53. Piontkewitz Y, Arad M, Weiner I. Abnormal trajectories of neurodevelopment and behavior following in utero insult in the rat. Biol Psychiatry. 2011;70:842-51.

54. Adam R, David AS. Patterns of anterior cingulate activation in schizophrenia: a selective review. Neuropsychiatr Dis Treat. 2007;3:87-101.

55. Gilabert-Juan J, Belles M, Saez AR, Carceller H, Zamarbide-Fores S, Moltó MD, et al. A 'double hit' murine model for schizophrenia shows alterations in the structure and neurochemistry of the medial prefrontal cortex and the hippocampus. Neurobiol Dis. 2013;59:126-40.

56. Alam M, Angelov S, Stemmler M, von Wrangel C, Krauss JK, Schwabe K. Neuronal activity of the prefrontal cortex is reduced in rats selectively bred for deficient sensorimotor gating. Prog Neuropsychopharmacol Biol Psychiatry. 2015;56:174-84.

57. Van Luijtelaar G, Fabene PF, De Bruin N, Jongema C, Ellenbroek BA, Veening JG. Neural correlates of sensory gating in the rat: decreased Fos induction in the lateral septum. Brain Res Bull. 2001;54:145-51.

58. Bannerman DM, Rawlins JNP, McHugh SB, Deacon RMJ, Yee BK, Bast T, et al. Regional dissociations within the hippocampus-memory and anxiety. Neurosci Biobehav Rev. 2004;28:273-83.

59. Pezze M, McGarrity S, Mason R, Fone KC, Bast T. Too little and too much: hypoactivation and disinhibition of medial prefrontal cortex cause attentional deficits. J Neurosci. 2014;34:7931-46.

60. McGarrity S, Mason R, Fone KC, Pezze M, Bast T. Hippocampal neural disinhibition causes attentional and memory deficits. Cereb Cortex. 2017;27:4447-62.

61. Río-Álamos C, Piludu MA, Gerbolés C, Barroso D, Oliveras I, Sánchez-González A, et al. Volumetric brain differences between the Roman rat strains: neonatal handling effects, sensorimotor gating and working memory. Behav Brain Res. 2019;361:74-85.
62. Woollett K, Maguire EA. Acquiring 'the knowledge' of London's layout drives structural brain changes. Curr Biol. 2011;21:2109-14.

63. Taubert $M$, Villringer $A$, Ragert $P$. Learning-related gray and white matter changes in humans. Neurosci. 2012;18:320-5.

64. Draganski B, Gaser C, Busch V, Schuierer G, Bogdahn U, May A. Changes in grey matter induced by training. Nature. 2004;427:311-2.

65. Bois C, Levita L, Ripp I, Owens DCG, Johnstone EC, Whalley HC, et al. Hippocampal, amygdala and nucleus accumbens volume in first-episode schizophrenia patients and individuals at high familial risk: a cross-sectional comparison. Schizophr Res. 2015;165:45-51.

66. Lazovic J, Wrzos HF, Yang QX, Collins CM, Smith MB, Norgren R, et al. Regional activation in the rat brain during visceral stimulation detected by c-fos expression and fMRI. Neurogastroenterol Motil. 2005;17:548-56.

67. Dodd GT, Williams SR, Luckman SM. Functional magnetic resonance imaging and c-Fos mapping in rats following a glucoprivic dose of 2-deoxy-d-glucose. J Neurochem. 2010;113:1123-32.

68. Swerdlow NR, Shoemaker JM, Bongiovanni MJ, Neary AC, Tochen LS, Saint Marie RL. Strain differences in the disruption of prepulse inhibition of startle after systemic and intra-accumbens amphetamine administration. Pharm Biochem Behav. 2007;87:1-10.

69. Alsene KM, Fallace K, Bakshi VP. Ventral striatal noradrenergic mechanisms contribute to sensorimotor gating deficits induced by amphetamine. Neuropsychopharmacology. 2010;35:2346-56.

70. Issy AC, Del Bel EA. 7-Nitroindazole blocks the prepulse inhibition disruption and c-Fos increase induced by methylphenidate. Behav Brain Res. 2014;262:74-83.

71. Palmer AA, Printz MP. Strain differences in Fos expression following airpuff startle in Spontaneously Hypertensive and Wistar Kyoto rats. Neuroscience. 1999;89:965-78.

72. Koch M. The neurobiology of startle. Prog Neurobiol. 1999;59:107-28. 